\title{
Extremal results for rooted minor problems
}

In this paper, we consider the following problem:

Given four distinct vertices $v_{1}, v_{2}, v_{3}, v_{4}$. How many edges guarantee the existence of seven connected disjoint subgraphs $X_{i}$ for $i=1, \ldots, 7$ such that $X_{j}$ contains $v_{j}$ for $j=1,2,3,4$ and for $j=1,2,3,4 \quad X_{j}$ has a neighbour to each $X_{k}$ with $k=5,6,7$. This is the so-called "rooted $K_{3,4}$-minor problem".

There are only few known results on rooted minor problems, e.g. [8,3]. In this paper, we prove that a 4 -connected graph with $n$ vertices and $5 n-14$ edges has

a rooted $K_{3,4}$-minor. Also, we consider the similar problems concerning rooted $K_{3,3}$-minor problem and $K_{3,2}$-problem. 